\section{High sensitivity cardiac troponin testing}

Bösner and colleagues have presented evidence for a simple rule for ruling out coronary artery disease in the primary care setting. ${ }^{1}$ It is noteworthy that no laboratory measurements have been included in their model, with reference to their introductory statement that " ... electrocardiography and cardiac troponin test are of limited value in primary care... " This point deserves some discussion as the utility of cardiac troponin testing appears to be expanding in step with increasing assay sensitivity. With high-sensitivity assays clinically available - an example being the high sensitivity cardiac troponin $\mathrm{T}$ ( $\mathrm{TnT}$ hs) assay now in use in Europe and available in Canada later this year, the ability to use this test in the primary setting for ruling out individuals at risk is an intriguing possibility. This is supported by recent studies in stable high- risk populations where the majority of individuals had measurable TnT hs concentrations, ${ }^{2,3}$ thus perhaps permitting interpreting $\mathrm{TnT}$ hs concentrations in the nonacute setting. More work is needed in this regard to establish cutoffs using the high-sensitivity assays in the primary care setting, but the ability to measure cardiac troponin in the stable setting is a provocative new route for possible risk stratification. Furthermore, even if these high sensitivity assays add prognostic information, an important question will remain whether inclusion of this marker to the existing five determinants will further improve an already impressive area under the curve of $0.90 .{ }^{1}$

\section{Peter A. Kavsak PhD}

Matthew J. McQueen MBChB PhD

Department of Pathology and Molecular Medicine, McMaster University, and the Population Health Research Institute, Hamilton, Ont.

Competing interests: Dr. Kavsak has received speaker fees and/or grant support from the following diagnostic companies: Beckman Coulter, Roche, Ortho Clinical Diagnostics, Randox Ltd. Dr. McQueen has received grant support from Roche and Beckman Coulter.
REFERENCES

1. Bösner S, Hassenritter J, Becker A, et al. Ruling out coronary artery disease in primary care: development and validation of a simple prediction rule. CMAJ 2010;182:1295-300.

2. Omland T, de Lemos JA, Sabatine MS, et al. A sensitive cardiac troponin $\mathrm{T}$ assay in stable coronary artery disease. N Engl J Med 2009;361:2538-47.

3. Kavsak PA, McQueen MJ. High sensitivity cardiac troponin concentration cutoffs - is a healthy population the right reference population for those with underlying cardiac disease? Clin Biochem 2010;43: 1037-8.

DOI:10.1503/cmaj.110-2110

\section{Incorrectly placed C-spine collar}

I don't question the research in this arti$\mathrm{cle}^{1}$ but rather the improper positioning of the C-spine collar on the cover of the Aug. 10 issue of CMAJ. The collar protrudes too far anteriorly (under the chin) and doesn't sit properly on the trapezius. This placement may result in serious injury if the chin slips under the collar during transport or a patient lift. An unfortunate portrayal of $\mathrm{C}$-spine to accompany a good article.

\section{Christopher J. Stephenson}

UBC MD Undergraduate, Vancouver, BC

\section{REFERENCE}

1. Stiell IG, Clement CM, O'Connor A, et al. Multicentre prospective validation of use of the Canadian $\mathrm{C}$-Spine Rule by triage nurses in the emergency department. CMAJ 2010 Aug 10;182:1173-9.

DOI:10.1503/cmaj.110-2121

\section{Medical education needed for smoking cessation}

The editorial by Penz and colleagues rightly argues that governments can improve the health of populations by paying for smoking cessation. ${ }^{1}$ Too often discussion of health care costs focuses on saving by reducing services to those who are sick, rather than by reducing the incidence of illness. Nevertheless, by concentrating on reimbursement for smoking cessation medications and products, Penz and colleagues ignore the need to pay for a more comprehensive approach to smoking cessation. All provinces have some tobacco control policies, such as restrictions on advertising and on smoking in public places, which help to reduce smoking rates.

But for smoking cessation, it is clear that advice and counselling on behavioural strategies is effective, alone or in combination with pharmacologic therapy. ${ }^{2}$ Provincial funding of smoking cessation must include reimbursement of physicians' time for counselling, as well as funding for other health professionals and programs to provide this service so that physicians do not carry this responsibility alone. However, physicians and other health professionals are not necessarily prepared by their education to provide smoking cessation counselling..$^{3,4}$ Medical education, and education of other health professionals, must include training in counselling for smoking cessation and other preventive health behaviour.

\section{Joan M. Brewster PhD}

Dalla Lana School of Public Health, University of Toronto, Toronto, Ont.

\section{REFERENCES}

1. Penz ED, Manns BJ, Hébert PC, et al. Governments, pay for smoking cessation. CMAJ 2010 Aug. 30. [Epub ahead of print]: $10.1503 / \mathrm{cmaj}$ .101140 .

2. Treating tobacco use and dependence: clinical practice guideline, 2008 update. Washington (DC) US Department of Health and Human Services, Public Health Service, 2008. Available: www surgeongeneral.gov/tobacco/treating_tobacco_use08 .pdf (accessed 2010 Oct. 6).

3. Richmond R, Zwar N, Taylor R, et al. Teaching about tobacco in medical schools: A worldwide study. Drug Alcohol Rev 2009;28:484-97.

4. Geller AC, Brooks DR, Powers CA, et al. Tobacco cessation and prevention practices reported by second and fourth year students at US medical schools. J Gen Intern Med 2008;23:1071-6.

DOI:10.1503/cmaj.110-2119

\section{Governments, pay for smoking cessation}

Your recent editorial correctly argues that provincial governments should provide funding for smoking cessation as it is a highly cost-effective way to improve population health. ${ }^{1}$ Alas, the editorial then repeats a common error of confusing cost-effectiveness with 